\title{
3D printed kidney phantoms for an LED-based photoacoustic and ultrasound imaging system
}

D. I. Nikitichev, K. Murawski, G. Harris, G. Perkins, S. Mosse, et al.

D. I. Nikitichev, K. Murawski, G. Harris, G. Perkins, S. Mosse, E. Maneas, W. Xia, V. Arumuham, Mithun Kunyuil Ajith Singh, S. Choong, A. Desjardins, J. Hebden, "3D printed kidney phantoms for an LED-based photoacoustic and ultrasound imaging system," Proc. SPIE 10878, Photons Plus Ultrasound: Imaging and Sensing 2019, 1087868 (27 February 2019); doi: $10.1117 / 12.2509831$

SPIE. Event: SPIE BiOS, 2019, San Francisco, California, United States 


\title{
3D Printed Kidney phantoms for an LED-based Photoacoustic and Ultrasound Imaging System
}

\author{
D. I. Nikitichev ${ }^{1}$, K. Murawski ${ }^{1}$, G. Harris ${ }^{1}$, G. Perkins ${ }^{1}$, S. Mosse ${ }^{1}$, E. Maneas ${ }^{1}$, W. Xia ${ }^{4}$, V. \\ Arumuham $^{2}$, Mithun Kuniyil Ajith Singh ${ }^{3}$, S. Choong ${ }^{2}$, A. Desjardins ${ }^{1}$, and J. Hebden ${ }^{1}$ \\ ${ }^{1}$ Department of Medical Physics Biomedical Engineering, University College London, Gower \\ Street, London WC1E 6BT, United Kingdom. \\ ${ }^{2}$ University College London Hospital, 235 Euston Road, London NW1 2BU, United Kingdom. \\ ${ }^{3}$ Research and Development Division, CYBERDYNE Inc. \\ ${ }^{4}$ School of Biomedical Engineering Imaging Sciences, Kings College London
}

\begin{abstract}
Photoacoustic imaging is a powerful and increasingly popular technique for tissue diagnostics. Suitable tissueequivalent phantoms are in high demand for validating photoacoustic imaging methods and for clinical training.

In this work, we describe a method of directly 3D printing a photoacoustic tissue-equivalent phantom of a kidney based on Gel Wax, which is a mix of polymer and mineral oil. A kidney phantom that is compatible with photoacoustic scanning will enable clinicians to evaluate a portable LED-based photoacoustic and ultrasound imaging system as a means of locating tumors and other abnormalities. This represents a significant step towards clinical translation of the compact system.

Training using realistic phantoms reduces the risks associated with clinical procedures. Complications during procedures can arise due to the specific structure of the kidney under investigation. Thus the ability to create a 3D printed phantom based on detailed anatomical images of a specific patient enables clinicians to train on a phantom with exactly the same structure as the kidney to be treated.

Recently we developed a novel 3D printer based on gel wax. The device combines native gel wax with glass microspheres and titanium dioxide $\left(\mathrm{TiO}_{2}\right)$ particles to obtain a medium with tissue-like optical and acoustic properties. 3D models created using this printer can be given a range of values of optical absorption reduced scattering coefficients.

The ability to 3D patient-specific phantoms at low cost has the potential to revolutionize the production and use of tissue-equivalent phantoms in future, and can be applied to a wide range of organs and imaging modalities.
\end{abstract}

Keywords: 3D-printing, tissue-mimicking material, kidney, phantom, gel-wax, photoacoustic, ultrasound

\section{INTRODUCTION}

Photoacoustic imaging is a rapidly emerging imaging modality which has a lot of potential benefits. It is able to derive images based off of the contrast of optical absorption coefficients data of tissue. As such, the use of a live, functional photoacoustic imaging technique is effective as it offers high specificity in differentiating between healthy and contrasted diseased tissue. ${ }^{1}$ Moreover this can be achieved at a spatial resolution significantly higher than other standard modalities such as ultrasound. Although it does not provide a higher penetration depth than ultrasound it photoacoustic imaging can provide composition information based on absorption information

Clinically, photoacoustic imaging has been demonstrated to be safe and effective when diagnosing diseased tissue while using tissue contrasting agents. It has also shown promise in other therapeutic applications such as drug-delivery ${ }^{2-4}$ and image-guided interventions and monitoring. ${ }^{5}$ In order to further successfully develop novel photoacoustic methods, it is crucial to create anatomically accurate and reproducible phantoms which will be paramount in validating novel photoacoustic imaging techniques.

Further author information: (Send correspondence to Daniil Nikitichev)

D. Nikitchev.: E-mail:, d.nikitichev@ucl.ac.uk

Photons Plus Ultrasound: Imaging and Sensing 2019, edited by Alexander A. Oraevsky, Lihong V. Wang, Proc. of SPIE Vol. 10878, 1087868 · C 2019 SPIE · CCC code: 1605-7422/19/\$18 · doi: 10.1117/12.2509831 
In recent years, 3D-printing technology has been increasingly applied in the fields of biotechnology and a wide range of other medical sciences. ${ }^{6-8}$ The technology offers the capability to form complex geometries by layering material with a sub-millimeter resolution, accurately recreating the form of computer aided design (CAD) models and other volumetric data. This offers potential capabilities in flexible rapid prototyping of complex forms and multi-material printing and the additive printing approach can reduce the developmental cost of manufacture by greatly reducing the material loss as well as simplifying fabrication as it permits production without the need of specific tools or the use of moulds. This is especially advantageous in the medical sector when patients increasingly rely on personalised treatments.

However, the materials used traditional and commercial 3D printing have most commonly been used for mechanical prototyping or form creation through FDM or some form of polymerisation. Thermoplastics such as PLA or ABS are currently widely used even in consumer fused deposition modelling (FDM) printers, with numerous other composites and materials available using more advanced, specialised printers for specific use. Although PVA is an available material which can be used for ultrasound and photoacoustic imaging ${ }^{9-12}$ its fabrication process can be time-consuming and unreliable whilst 3D-printing. Moreover, it doesn't offer a tissue mimicking texture as it has a high Young's modulus. Most other plastics and resins have have unrealistic sound speeds and high acoustic attenuation.

For the modality of photoacoustic imaging, it is important to manipulate the phantoms optical properties, such as diffusion and absorption coefficients, as well as the acoustic properties of acoustic attenuation and impedance. A kidney model for photoacoustic imaging and validation would need to be made of a tissuemimicking material (TMM) which simulates the photoacoustic properties of kidney tissue. The results of studies characterizing the properties of the oil-based gel-wax, mixed with several additives, as an optical and ultrasound TMM have demonstrated its suitability as photoacoustic TMM. ${ }^{13,14}$ Gel-wax is widely used in the candle industry and has been recntly used for optical imaging and needle biopsy phantoms. ${ }^{15-17}$ Moreover, an approach can be used using gel-wax to adjust its photoacoustic properties by varying the concentration of its additives. Titanium Oxide $\left(\mathrm{TiO}_{2}\right)$ is used to tune optical scattering properties and glass microspheres are been used to tune and mimick tissue acoustic properties. The inclusion of carbon black dye, which is used for providing optical contrasting, mimicking contrasted diseased tissue, is known to have minimal effect on the acoustic properties of the gel-wax mixture. Thus, the additives may be used in conjunction to finely tune the TMM's photoacoustic properties.

Combining the advantages and desirable qualities of the gel-wax-based TMM and 3D printing allows the rapid manufacture of geometrically and anatomically accurate phantoms. Using techniques such as multi-material extrusion can increase the complexity of such models to allow for the creation of phantoms with varying optical and acoustic contrasts printed within the same structure. One such application studied here is embedding a cancerous lesion into the phantom for validation of photoacoustic systems.

\section{METHODOLOGY}

\subsection{Process Flow}

One of the overall aims of the study is to develop a proof of concept to demonstrate the feasibility of creating a patient-specific photoacoustic phantom with potential clinical use. A standard process of its manufacture, starting from clinic was developed for use in the study. 


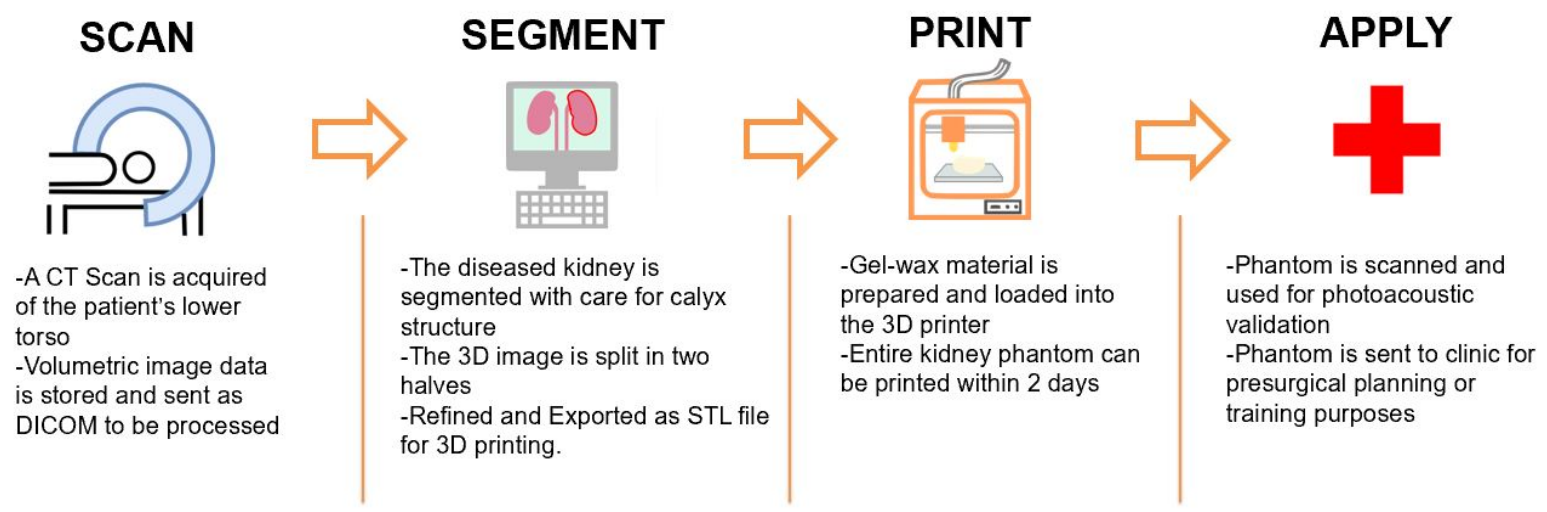

Figure 1. Graphical Process Flowchart, summarizing study objectives

In summary, this process involves obtaining patient data of the lower abdomen, from which DICOM data must be processed to extract the volumetric data of the kidney. The geometry is then refined for 3D printing. It is then rapidly manufactured using a specialised gel-wax 3D printer and delivered back to clinic for use. Success using this approach would allow for very rapid patient-to-clinic phantom creation for applications such as pre-surgical planning and training.

\subsection{Data Extraction and Refinement}

Patient DICOM data from a CT scan (PROVIDED BY X) of the lower abdomen was used to extract the 3D form of the kidney. The kidney was first segmented using freely available segmentation tools (Seg3D).
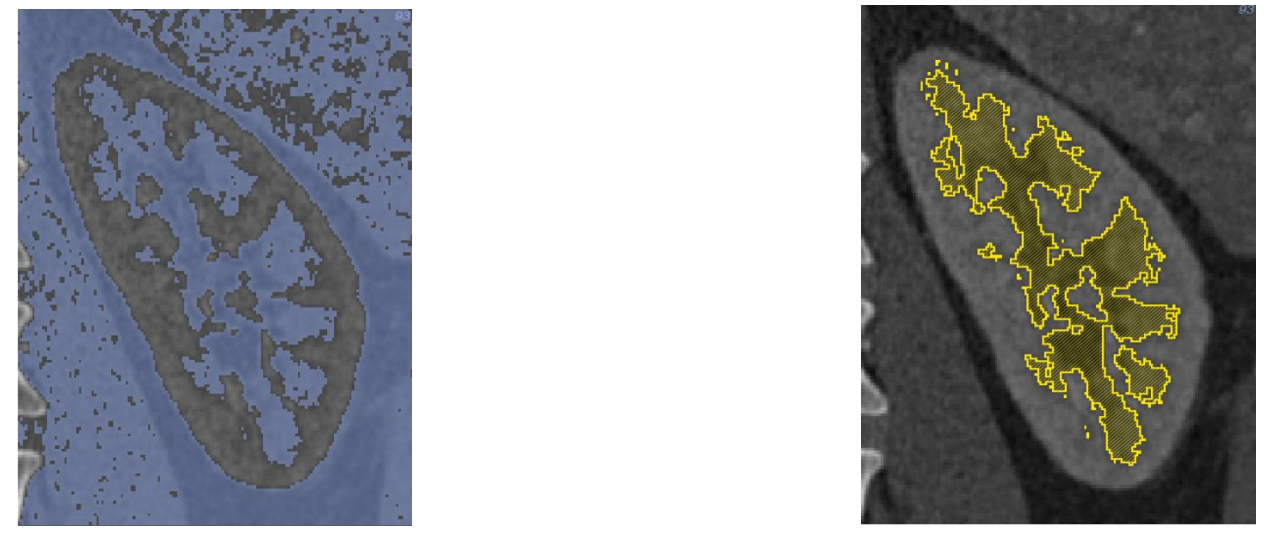

Figure 2. Sagittal Slice in Seg3D of kidney segmentation (left) and calyx separation (right)

A threshold was first applied to segment the kidney's external structure from its surrounding tissue and internal calyx structure (pictured in blue in Figure 22). Once the entire kidney was segmented, the region of the internal structure (highlighted in yellow) was cut from the 3D space.

Boolean filtering was used to remove any noise proximal to the perimeter of the calyxes. An intersectional mask was applied to remove the outlying voxels. Artefacts were carefully manually excluded whilst paying attention to retaining the detail of the calyces.

The segmented 3D form was exported to a mesh (as an STL file). The lower resolution of the data that produced the resulting STL file contained visible voxel aliasing. A smoothing function was used in Meshmixer by applying a 'shape preserving' filter, using the parameters of smoothing scale $=4$, constraint rings $=3$. Inspection was conducted to remove any geometric errors and holes were filled using a 'smooth-fill' function with threshold $0.01 \mathrm{~mm}$. 
In order to prime the kidney model for printing, Blender was used to slice the kidney into two separate files to be printed individually. This was done so that the kidney model can be printed without the need of generating support structures which when printing with gel wax, can interfere with the integrity of its shape.

The two STL files were then scanned and repaired using Netfabb to prevent any errors or breakages in the final STL geometry which may result in errors while exporting to g-code and printing.

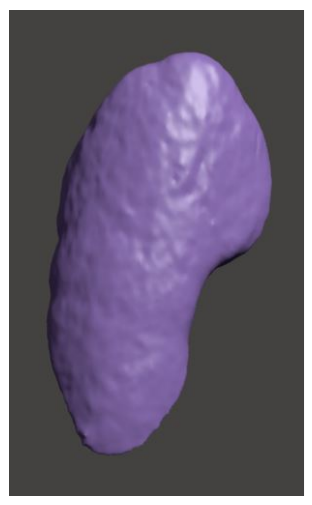

Back

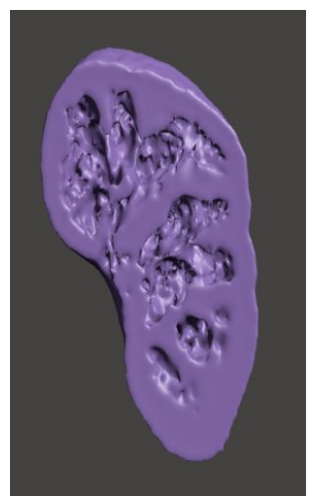

Front

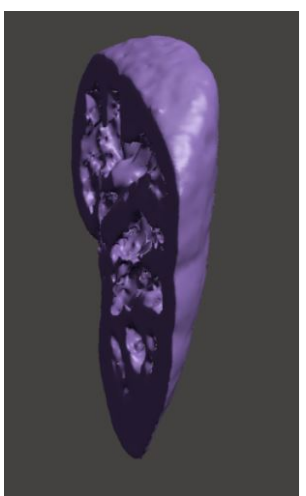

$45^{\circ}$ Rotation

Figure 3. Rendered STL file of half of the kidney

The STL files were opened in Slic3r to be exported into g-code. This allows the 3D file to be converted into an instruction set for the 3D printer. Furthermore settings including the print speed and nozzle temperature were set to ensure optimal print quality. This is achieved lowering the gel-wax viscosity enough to be extruded well at the nozzle tip, as well as adjusting the speed which allows the gel-wax mixture to settle consistently.

\subsection{Material Preparation}

Material to mimic healthy tissue within the kidney is made of a mixture of gel-wax containing $0.1 \% \mathrm{v} / \mathrm{v} \%$ Titanium oxide $\left(\mathrm{TiO}_{2}\right)$ and $0.5 \% \mathrm{w} / \mathrm{w} \%$ glass microspheres.

The inclusion of glass microspheres introduces dispersed particlates which modulate the acoustic attenuation properties of the gel-wax mixture. In a similar fashion, Titanium oxide provides the medium for optical scattering.

This mixture was achieved through melting the gel-wax and diffusing the added materials through a process of mixing and ultrasound sonication, to ensure the breaking up of the particulates. This process was repeated three times. The mixture is then degassed to prevent the formation of air bubbles which can affect the acoustic properties of the phantom.

Unhealthy, cancerous tissue was prepared in the same way as healthy tissue with \% Titanium oxide (TiO2) and $0.5 \% \mathrm{w} / \mathrm{w} \%$ glass microspheres and $0.05 \%, 0.1 \%, 0.25 \%$ and $0.5 \% \mathrm{w} / \mathrm{v} \%$ carbon black dye.

\section{$2.43 \mathrm{D}$ printing}

The 3D printer designed for printing gel-wax has a triple extrusion system, allowing it to print 2 different types of gel wax, as well as a PLA extrusion system. This allows for the development of more complicated prints that could be used for printing both unhealthy and healthy tissue.

The prepared gel-wax sample of healthy tissue was loaded into the 3D-printing system. By using the Kidney's 3D data, its geometry was printed by extruding the gel-wax at a steady rate, using a layer-by-layer modelling approach.

The whole kidney was printed in two parts, each as a half, sliced along the kidneys anatomical sagittal plane. The print was oriented so that the flat, sliced section was aligned with the print-bed. This is done so that the layers will be deposited in a stable configuration, with the least chance of misprinting caused by the lack of support structures (such as mid-air extrusion). This method is used to enhance the accuracy in regard to printing the calyx structures and maintain its $3 \mathrm{D}$ integrity. 

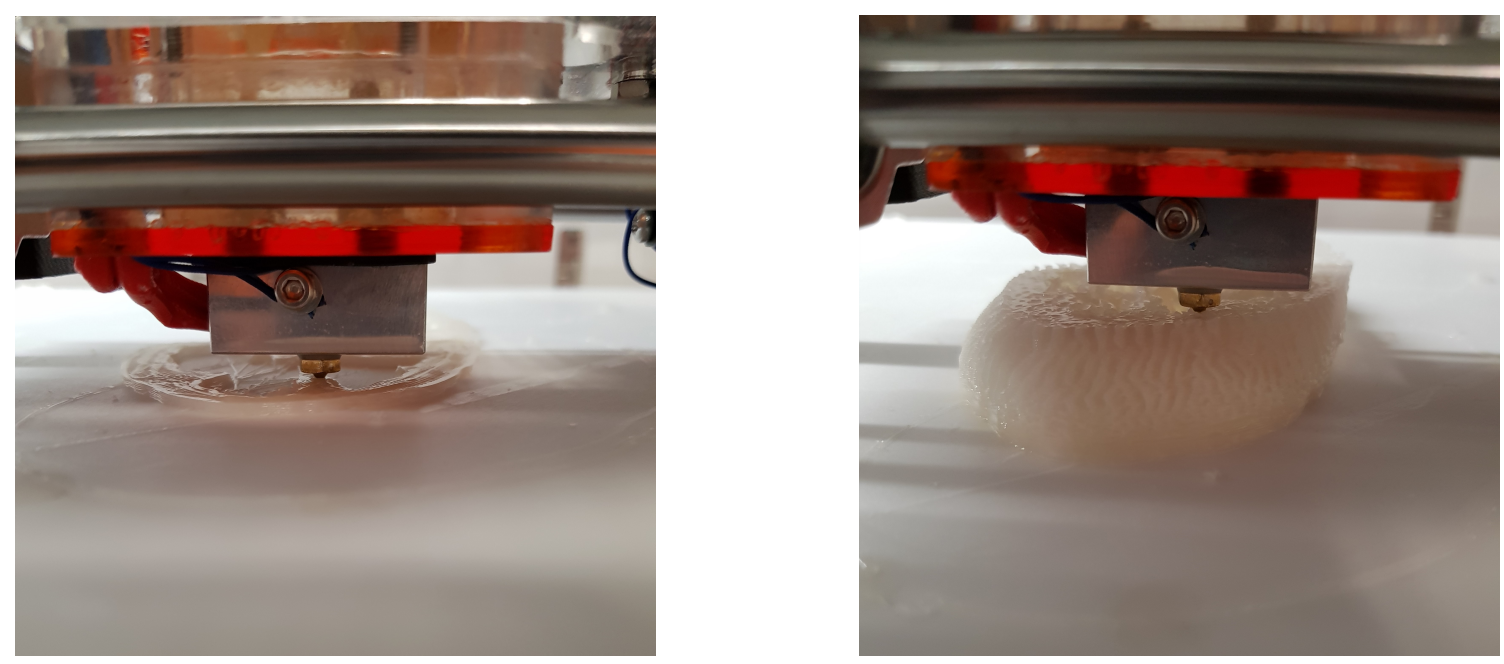

Figure 4. View of printhead at two points during mid-print

\subsection{Photoacoustic scanning and validation}

The completed phantom was scanned using the Cyberdyne Acoustic X LED-based photoacoustic system. Small slices of $0.5 \times 0.5 \times 0.2 \mathrm{~cm}$ diseased tissue-mimicking gel-wax samples were placed inside two positions, A and B in the kidney and images were acquired at both insertion points. The location of the simulated lesion was found by scanning the phantom whilst maneuvering the probe in real-time with a high refresh-rate.

$2 \mathrm{D}$ images with a maximum depth of $4 \mathrm{~cm}$ were acquired using a $850 \mathrm{~nm}$ wavelength and a $9 \mathrm{Mhz}$ ultrasound transducer. The visibility of the lesion due to optical contrast was observed. The images required both display an overlay of the photoacoustic image superimposed over the ultrasound image. The data was used to gauge the similarity of the phantoms acquired images to patient data.

\section{RESULTS}

\subsection{Preliminary Attempts}

Several iterations were printed of the kidney's top-half using gel-wax with $0.1 \% \mathrm{v} / \mathrm{v} \%$ Titanium Oxide and $0.5 \% \mathrm{v} / \mathrm{v} \%$ glass microspheres with varying print settings to optimize the quality of the print.

The final kidney was printed with a fluid and stable gel-wax viscosity at a print-speed which allowed for a total manufacture time of under 42 hours. Proper adhesion between gel-wax layers was ensured without losing structural integrity due to heat deformation. Gel-wax consistently filled out the spatial volume required to remove spaces that may form due to under-extrusion, yet without over-extruding from under-depositing gel-wax. 

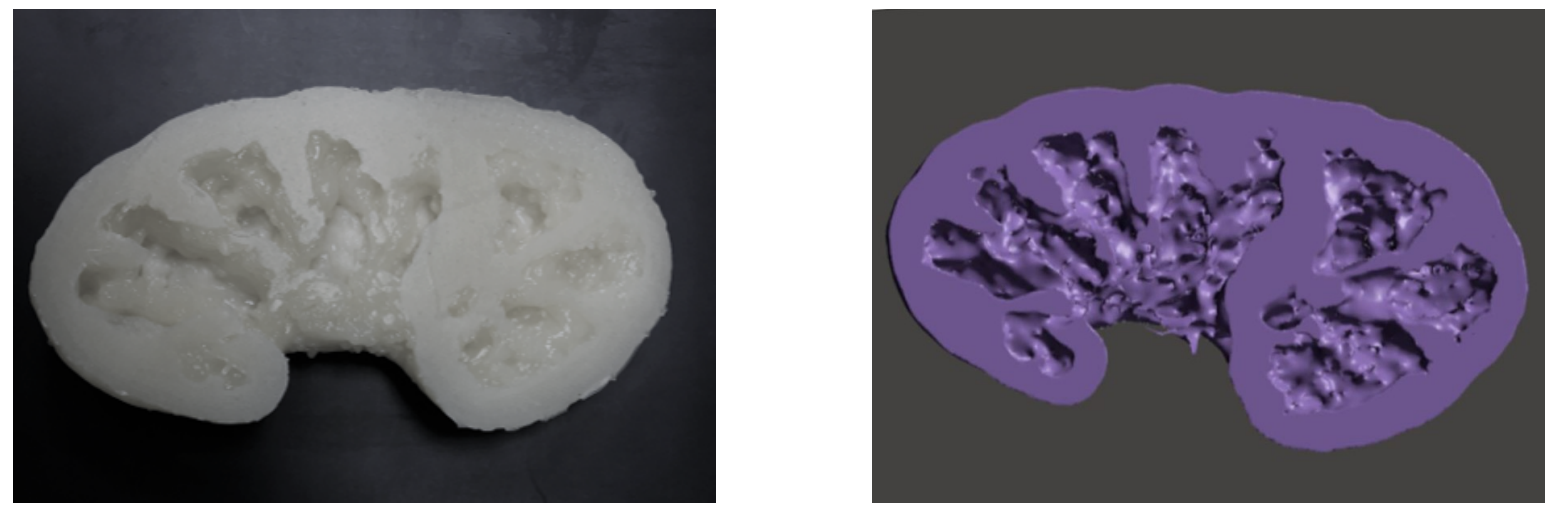

Figure 5. Comparison of unused preliminary print with 3D mesh showing structural similarity in printed calyx structures

The print resulted with a high-quality geometric replication of the 3D model when concerning the complex calyx formations.

\subsection{D Print}

The final 3D printed model displayed a high level of geometric similarity to the CAD 3D model. On further inspection, the consistency in extrusion rate appeared to be sub-optimal, resulting in slight deformations in the calyx region.

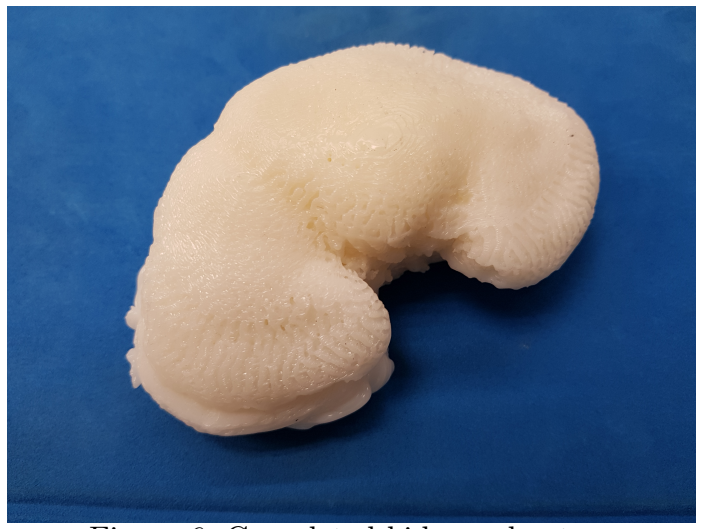

Figure 6. Completed kidney phantom

The total collective print-time lasted less than 43 hours, demonstrating feasibility in for use in rapid prototyping in patient specific applications, such as pre-surgical planning in percutaneous nephrolithotomy.This is assuming that the kidney segmentation from patient-data has been pre-prepared.

\subsection{Photoacoustic and ultrasound imaging}

The results of the photoacoustic images clearly show the phantoms success at mimicking a kidneys photoacoustic properties. The majority of attempts using the ultrasound/photoacoustic system at a depth of approimately $3-4 \mathrm{~cm}$ to find the sample of diseased TMM were successful 


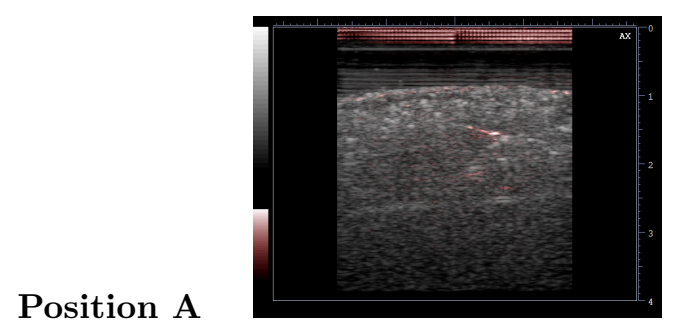

$0.05 \%$

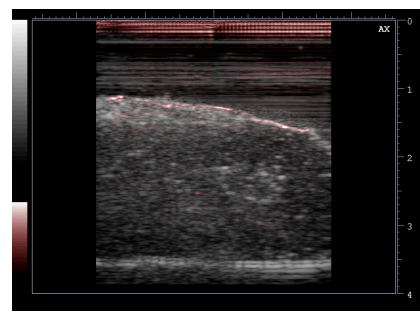

$0.05 \%$

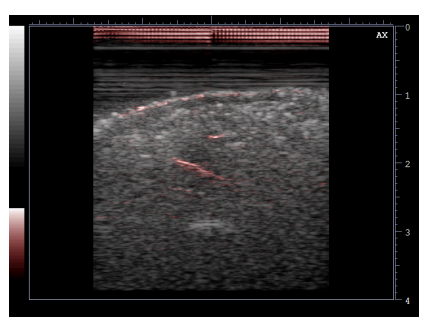

$0.1 \%$

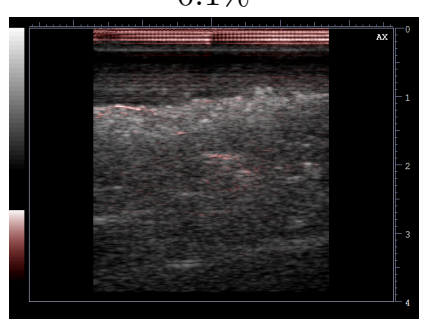

$0.1 \%$

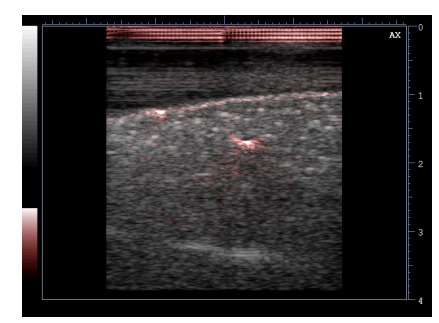

$0.5 \%$

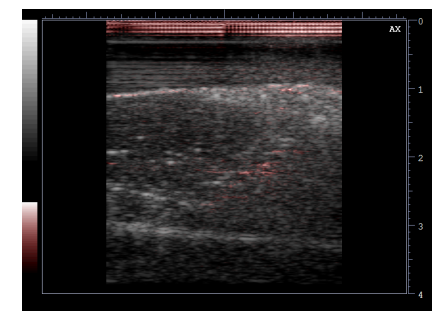

$0.5 \%$

Figure 7 . Images of unhealthy tissue (0.05\% Carbon black dye) embedded within the 3D printed Kidney

The above images acquired by a photoacoustic/ultrasound system using a low optical contrast from the sample of gel-wax mixture with $0.05 \%$ Carbon black dye was difficult to acquire. This is because the signals received from the unhealthy tissue was very weak. It would be unlikely that this optically contrasting body would have been found if the its original position was unknown. Nevertheless the signal was only visible in the case of Position A. As expected, no significant difference in acoustic attenuation was observed in the ultrasound scan.

Similarly, it appears as though the addition of $0.05 \%$ carbon black dye remains on the borderline of acceptability. This is because while it displays good optical contrast, and the imaging system receives strong signalling, pinpointing its location with ease, it appears to be too easily spotted to have been a realistic phantom of an in-vivo scan.

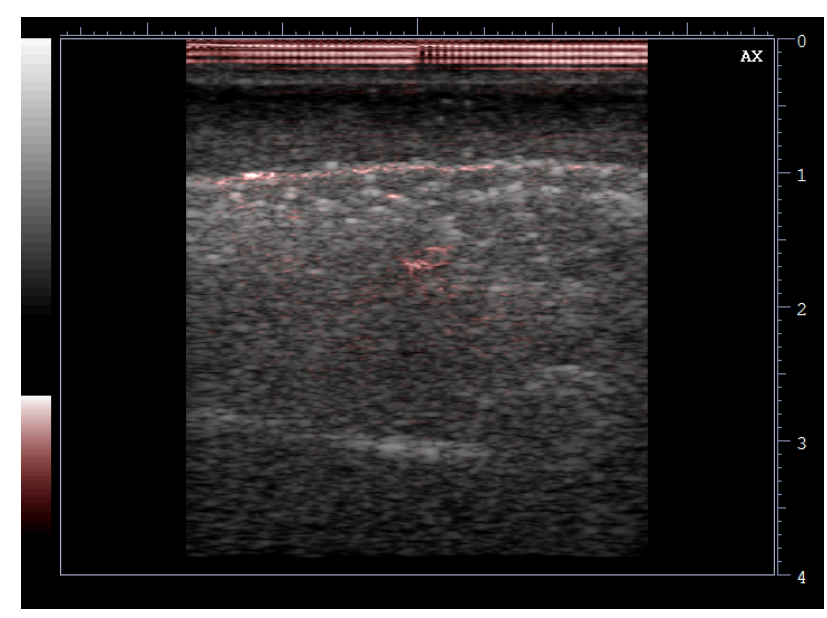

$0.05 \%$ Position A

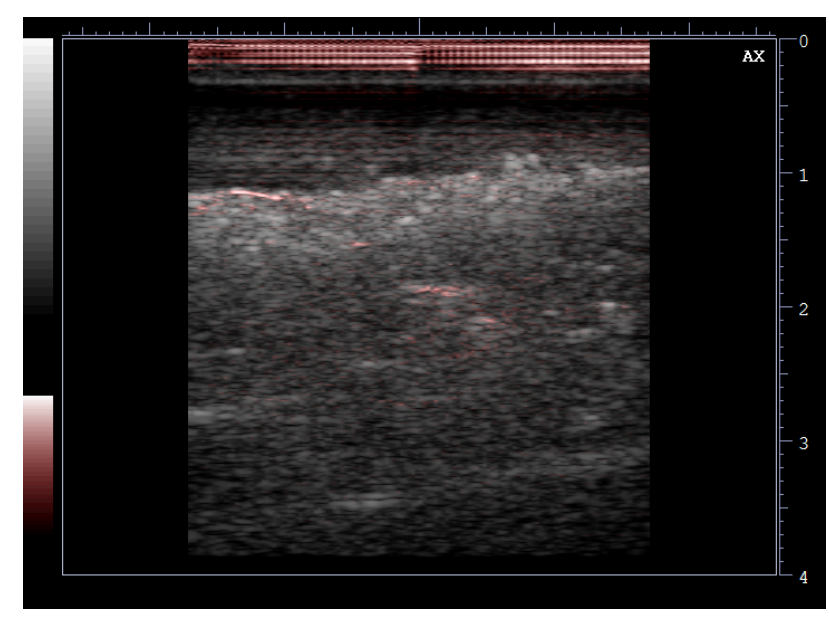

$0.05 \%$ Position B

Figure 8. Images of diseased TMM embedded within the 3D printed Kidney phantom 


\section{CONCLUSION}

The study conducted details the first successfully 3D-printed TMM kidney model for photoacoustic testing. Although the optimization of the 3D printing technology for the rapid prototyping of kidney-like structures made from gel-wax is at its infancy, the results demonstrate viability in this technique. Nevertheless, the study has achieved its goal of developing an inexpensive and rapidly produced multi-modal kidney phantom for ultrasound and photoacoustic applications.

The process used widely available, inexpensive materials, as well as ubiquitous 3D printing technology, which demonstrates the potential to increase cost-effectiveness and efficiency in pre-surgical planning as well as for training purposes. These advantages in the technology's ease of access can potentially facilitate this technique's widespread use in the future.

\section{ACKNOWLEDGMENTS}

This work is supported by the Wellcome/EPSRC Centre for Interventional and Surgical Sciences (WEISS) $(203145 \mathrm{Z} / 16 / \mathrm{Z})$.

\section{REFERENCES}

[1] Beard, P., "Biomedical photoacoustic imaging," Interface Focus (2011).

[2] Mallidi, S., P.Luke, G., and Emelianov, S., "Photoacoustic imaging in cancer detection, diagnosis, and treatment guidance," Trends in Biotechnology (2011).

[3] Valluru, K. S. and Willmann, J. K., "Clinical photoacoustic imaging of cancer," Ultrasonography (2016).

[4] Su, J. L., Wang, B., Wilson, K. E., Bayer, C. L., Chen, Y.-S., Kim, S., Homan, K. A., and Emelianov, S. Y., "Advances in clinical and biomedical applications of photoacoustic imaging," Expert Opin Med Diagn 4(6) (2010).

[5] Xi, L., Zhou, G., Gao, N., Yang, L., Gonzalo, D. A., Hughes, S. J., and Jiang, H., "Photoacoustic and fluorescence image-guided surgery using a multifunctional targeted nanoprobe," Annals of Surgical Oncology (2014).

[6] Rengier, F., Mehndiratta, A., von Tengg-Kobligk, H., Zechmann, C. M., Unterhinninghofen, R., Kauczor, H.-U., and Giesel, F. L., "3d printing based on imaging data: review of medical applications," International Journal of Computer Assisted Radiology and Surgery (2010).

[7] Marro, A., Bandukwala, T., and Mak, W., "Three-dimensional printing and medical imaging: A review of the methods and applications," Current Problems in Diagnostic Radiology .

[8] Martelli, N., Serrano, C., van den Brink, H., Pineau, J., Prognon, P., Borget, I., and Batti, S. E., "Advantages and disadvantages of 3-dimensional printing in surgery: A systematic review," Surgery .

[9] Surry, K. J. M., Austin, H. J. B., Fenster, A., and Peters, T. M., "Poly(vinyl alcohol) cryogel phantoms for use in ultrasound and mr imaging," Physics in Medicine and Biology (2004).

[10] Kharine1, A., Manohar1, S., Seeton, R., Kolkman, R. G. M., Bolt, R. A., Steenbergen, W., and de Mul, F. F. M., "Poly(vinyl alcohol) gels for use as tissue phantoms in photoacoustic mammography," IOP (2003).

[11] Kolkman, R. G. M., Hondebrink, E., Steenbergen, W., van Leeuwen, T. G. J. M., and de Mul, F. F. M., "Photoacoustic mammography laboratory prototype: imaging of breast tissue phantoms," J. Biomed. Opt.

[12] S, M., A, K., van Hespen J.C.G, W, S., and van Leeuwen T. G., "Photoacoustic mammography laboratory prototype: imaging of breast tissue phantoms," J. Biomed. Opt. 9(6) .

[13] Maneas, E., Xia, W., Ogunlade, O., Fonseca, M., Nikitichev, D. I., L, A., David, S. J. W., Ourselin, . S., Hebden, J. C., and Tom Vercauteren, A. E. D., "Gel wax-based tissue-mimicking phantoms for multispectral photoacoustic imaging," Biomed Opt Express (2018).

[14] Maneas, E., WenfengXia1, Nikitichev, D. I., Daher, B., Manimaran, M., Wong, R. Y. J., Chang, C.-W., Rahmani, B., Capelli, C., Schievano, S., Burriesci, G., Ourselin, S., David, A. L., Finlay, M. C., West, S. J., Vercauteren, T., and Desjardins, A. E., "Anatomically realistic ultrasound phantoms using gel wax with 3d printed moulds," Physics in Medicine and Biology (2018). 
[15] L, V. S., Z, P. T., E, J. J., and O, C. A. A., "Paraffin-gel tissue-mimicking material for ultrasound-guided needle biopsy phantom," Ultrasound Med. Biol. 39(12) (2013).

[16] X, X. R., J, E., H, E.-D., B, W., and P., P. S., "Design and benchtop validation of a handheld integrated dynamic breast imaging system for noninvasive characterization of suspicious breast lesions," Technol. Cancer Res. Treat. $7(6)$.

[17] E, D., Z, Z., M, W., Y, X., S, L., P, S., L, C., and X, X. R., "Three-dimensional fuse deposition modeling of tissue-simulating phantom for biomedical optical imaging," J. Biomed. Opt. 20(12) . 